\title{
Miniaturisierter energieautarker binärer Sensor auf Basis von feuchtigkeitsempfindlichen Hydrogelen
}

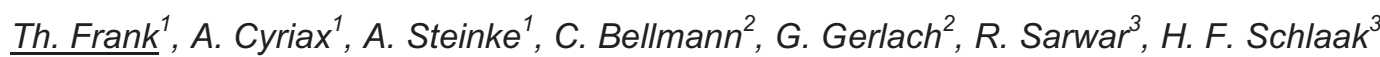 \\ ${ }^{1}$ CiS Forschungsinstitut für Mikrosensorik und Photovoltaik GmbH, 99099 Erfurt, Deutschland \\ tfrank@cismst.de \\ 2 Technische Universität Dresden, Institut für Festkörperelektronik, 01069 Dresden, Deutschland \\ ${ }^{3}$ Technische Universität Darmstadt, Fachgebiet Mikrotechnik und Elektromechanische Systeme, \\ 64283 Darmstadt, Deutschland
}

\begin{abstract}
Kurzfassung
Dieser Artikel präsentiert ein Konzept zum energieeffizienten Betreiben autonom vernetzter Sensornetzwerke. Diese benötigen für einen 24/7 Betrieb ein angepasstes Energiemanagement. Durch die entwickelten leistungslosen binären Mikroschalter wird ein eventabhängiges Einschalten des jeweiligen Sensorknotens erreicht. Die Plattform ist für unterschiedliche Messgrößen geeignet. Die direkt aus der Messgröße gewonnene mechanische Energie schaltet im einfachsten Fall mechanisch zwei elektrische Kontakte. Die Funktion wir hier am Beispiel eines realisierten Schwellwertschalters für die relative Luftfeuchtigkeit der Umgebungsluft dokumentiert. Als sensitives Material wird ein Hydrogelgemisch aus PVA und PAA verwendet. Zur Generierung der Schaltbewegung wirkt das aktive Hydrogel zusammen mit einer passiven Siliziumbiegeplatte als sogenanntes Unimorph verwendet. Das Schaltelement wird aus einer Glasgrundplatte mit Kontaktflächen aus Gold-Nanodrähten realisiert. Die zum Schalten benötigte Energie bezieht der Mikroschalter aus dem Umgebungsmedium. Diese Energie wird in eine Bewegung und dann in einen Schaltzustand umgesetzt. Die auf diese Weise generierte mechanische Arbeit schließt oder öffnet einen elektrischen Kontakte, in Abhängigkeit von der entsprechenden definierten Messgröße. Der große Vorteil dieses binären Mikroschalters besteht darin, dass er vollkommen auf elektrische Energie verzichten kann.
\end{abstract}

Schlüsselwörter: binärer Sensor, binärer Mikroschalter, energieautark, Siliziumbiegeplatte, Hydrogel, (PVA/PAA)

\section{Einleitung}

Die Prozessregelung von nahezu $70 \%$ aller technischen und nicht technischen Prozesse erfolgt auf Basis von Grenzwertparametern. Nahezu die gesamte Sensorik in der Gebäudeautomatisierung basiert auf der Zweipunktregelung. Die generelle Zielstellung ist es, diese Sensorsysteme energiesparsam oder im Idealfall energieautark zu realisieren. Energieautark sind Sensoren, wenn sie aus dem aktuellen Umfeld mit Energie versorgt werden. Beim Energie Harvesting wird dazu die Umgebungsenergie in elektrische Energie gewandelt. Eine wichtige Anwendung ist die Verwendung in autonomen Sensornetzwerken. Nachteilig an dieser Struktur ist, der hohe Verbrauch an elektrischer Energie für einen 24/7 Einsatz (Abb. 1). Um Energie zu sparen, besteht nun die Möglichkeit, die Elektronik in einen Schaltzustand zu versetzen, wo diese keine elektrische Energie verbraucht, (Power-Off). Möglich ist auch der Standby Zustand. Nachteilig ist, dass die Sensoren der Sensorknoten in diesem
Zustand nicht aktiv sind. Viele dieser Sensorknoten dienen dazu, ein großes Areal zu überwachen und bei Eintritt eines ungewöhnlichen Ereignisses dieses zu melden, welche naturgemäß eher selten stattfinden sollten, Beispiele sind Feuer-, Rauch- oder Feuchtemelder.

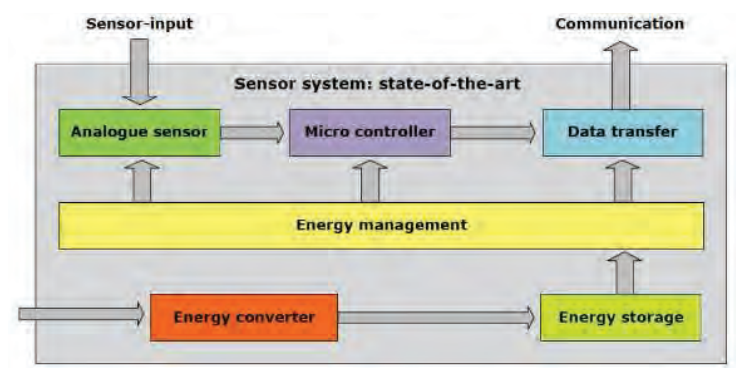

Abb. 1: Signalsystem -- Stand der Technik (ständig online)[1]

Mit einer neuen Struktur lässt sich dieser Nachteil bei gleicher Energieeffizienz beseitigen. Basis dieser Struktur ist ein sensitiver Mikroschalter, (BIZEPS Binary Zero Power Sensor). Dieser sensitive Mikroschalter schaltet in Ab- 
hängigkeit einer Messgröße das System online. Abb. 2 zeigt die Struktur. Das System ist im Normalzustand aus (Power-Off), bei Eintritt eines Ereignisses schaltet der Mikroschalter abhängig vom Event das System ein. Der Sensorknoten kann nun zum Beispiel durch Verwendung weiterer Sensoren überprüfen ob es sich um ein relevantes Ereignis handelt und dieses zur Basis melden.

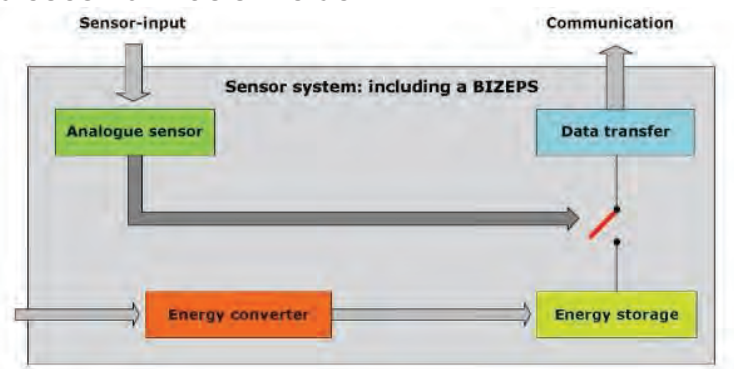

Abb. 2: Signalsystem -- binärer Mikroschalter (ereignisabhängig online)[1]

\section{Sensorische Eigenschaft}

Der Mikroschalter bezieht die zum Schalten benötigte Energie aus dem Messmedium. Dazu finden Funktionsmaterialien Verwendung, die sehr sensitiv und definiert auf die Änderung von Umweltgrößen mit einer Form- oder Volumenänderung reagieren. Die auf diese Weise generierte mechanische Arbeit schließt oder öffnet einen elektrischen Kontakt, in Abhängigkeit von der entsprechenden vordefinierten Messgröße (Abb. 3). Der große Vorteil dieses Mikroschalters besteht darin, dass sie vollkommen auf elektrische Energie verzichten.

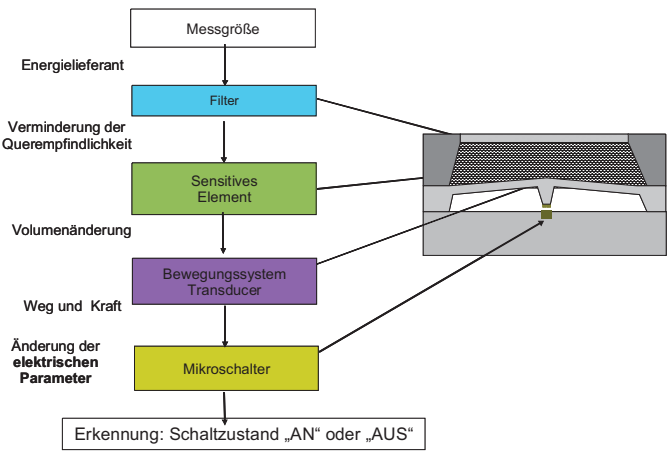

Abb. 3: vereinfachtes Prinzip binärer Mikroschalter

Im vorliegenden Anwendungsfall wird ein Hydrogel verwendet, welches in Abhängigkeit der relativen Umgebungsfeuchte quillt. Diese Energie, hervorgerufen durch die Volumenvergrößerung, wird in eine Bewegung und dann in einen Schaltzustand umgesetzt.

Das verwendete Hydrogel ist eine Mischung aus 15 Gew.\% Poly(vinylalkohol) (PVA) und 7,5 Gew.\% Poly(acrylsäure) (PAA).

\section{Bewegungssystem}

Die Aufgabe des Bewegungssystems ist die Wandlung der Reaktion des sensitiven Elementes in eine Schaltbewegung und eine Kontaktkraft. Hierfür kann die 3-dimensionale Volumendehnung direkt durch einen abgeschlossenen Raum, welcher bis auf einen nachgiebigen Bereich steif ist, genutzt werden. Dieses Prinzip ermöglicht zwar eine hohe Schaltkraft, die Befüllung gestaltet sich aber sehr aufwendig. Technologisch einfacher zu realisieren ist ein Wandler, der als Unimorph arbeitet. In der Abb. 4 sind diese beiden Effekte schematisch dargestellt. Zur Evaluierung wurde ein Unimorph, bestehend aus einer $20 \mu \mathrm{m}$ dicken passiven Siliziumbiegeplatte und dem aktiven Hydrogel aufgebaut.

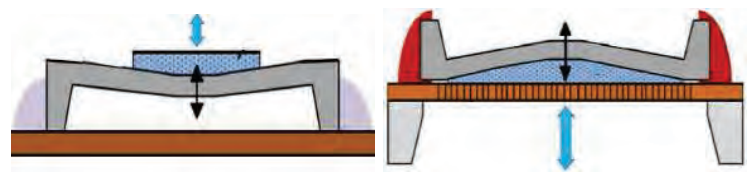

Abb. 4: Prinzip des Unimorph-Effekts (links) und des Volumeneffekts (rechts)

Die experimentellen Ergebnisse zeigten, dass beide Effekte ausreichend Auslenkungen der Siliziumbiegeplatte ermöglichen, beim unimorphen Effekt bis zu $23 \mu \mathrm{m}$ und $36 \mu \mathrm{m}$ bei der Volumenquellung. Eine optimale und nahezu lineare Korrelation zwischen der relativen Luftfeuchtigkeit und der Auslenkung wurde für die unimorph Wirkung aufgezeigt (Abb. 5). Der geschätzte Quellungsdruck betrug $38 \mathrm{kPa}$ in einem Bereich von 10 bis $85 \%$ r. $\mathrm{H}$.

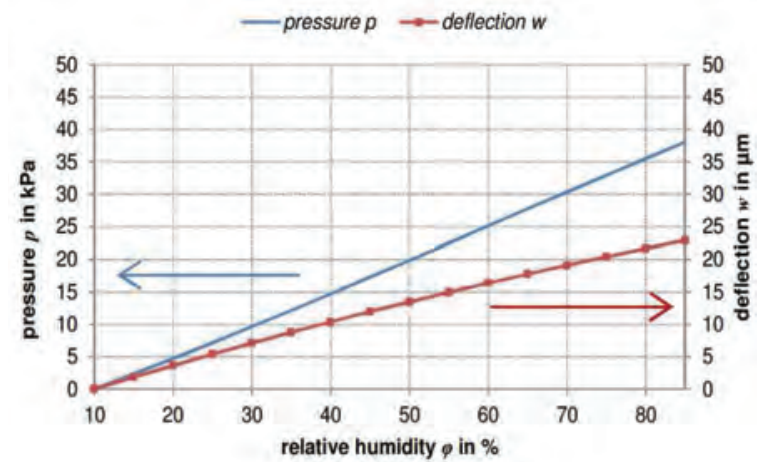

Abb. 5: Auslenkung beim unimorphen Effekt

Die Untersuchungen zum Quellungsdruck des unimorphen Wandlers und der daraus resultierenden Durchbiegung wurde mit dem in Abb. 6 dargestellten Prototyp durchgeführt. Um den Quellungsdruck abschätzen zu können wurde über den Anschluss ein Luftdruck $p$ angelegt der mechanisch die gleiche Wirkung auf die Biegeplatte hat, wie das Hydrogel. 


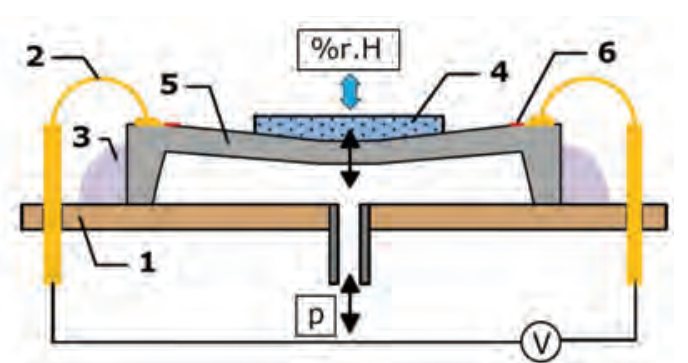

Abb. 6: Aufbau des Prototyps zur Quellungsmessung des unimorphen Effekts (1. TO8-Buchse, 2. Gold-Draht-Bindung, 3. Klebstoff, 4. PVA/PAASchicht, 5. Silizium-Chip, 6. Dehnungsmessstreifen)

Die Untersuchung zeigt, dass der Unimorph sowohl eine genügend große Kontaktkraft, als auch eine genügende Auslenkung aufweist.

\section{Mikrokontakt}

Das Schaltelement ist ein weiteres wichtiges Element. Zum Schalten elektrischer Kontakte unter Last sind einige Kriterien zu erfüllen.

- Eine ausgeprägte Schaltcharakteristik für die Vermeidung undefinierter Schaltzustände (Schnappeffekt)

- Ausreichende Kontaktkraft für einen geringen Übergangswiderstand

- Mechanisch und elektrisch belastbare Kontaktmetalle

- Kein Verschweißen der Kontakte durch die Belastung des Schaltvorgangs

Für feinwerktechnisch hergestellte Relais wird häufig Hartgold verwendet. Für einen Kontaktwiderstand im mOhm-Bereich werden Kontaktkräfte im $\mathrm{mN}$-Bereich benötigt.

Aufgrund der zu erwartenden geringen Kontaktkräfte $(<1 \mathrm{mN})$ durch das dynamische Verhalten der Hydrogelschicht, muss ein besonderes Design der Kontaktfläche angewandt werden. Der Mikrokontakt soll bereits bei Kontaktkräften von einigen $100 \mu \mathrm{N}$ sicher arbeiten. Basismodel für den Kontaktwiderstand ist die Hertzschen Pressung (1). Die für den Widerstandmaßgebliche Größe ist die Berührungsfläche, diese ist bei zwei elastischen Körper von der Geometrie, den Materialparametern und der Kontaktkraft abhängig.

Am Beispiel der Punktberührung Kugel - Kugel (1) ist leicht nachzuvollziehen, dass bei geringer Kontaktkraft $F$, großem Radius $r$ die Hertzschen Pressung $p_{\max }$ klein wird, was auch zu einer kleinen Kontaktfläche und zu einem hohem Übergangswiderstand führt.

$p_{\max }=\frac{1}{\pi} \cdot \sqrt[3]{\frac{1,5 \cdot F E^{2}}{r^{2}\left(1-\nu^{2}\right)^{2}}}$

$$
\begin{aligned}
& \text { und } \begin{aligned}
r=\frac{r_{1} r_{2}}{r_{1}+r_{2}} \\
\text { und } \quad E=2 \frac{E_{1} E_{2}}{E_{1}+E_{2}}
\end{aligned}
\end{aligned}
$$

mit

v: Geometrisches Mittel der Poissonzahl Körper 1, Körper 2

E: E-Modul der Werkstoffe Körper 1, Körper 2

$r$ : Radius von Körper 1, Körper 2

In der Abb. 7 (links) ist ein herkömmliches Kontaktpaar dargestellt. Bei geringer Kontaktkraft werden die Rauhspitzen nicht wesentlich eingeebnet. Der Kontaktwiderstand ist somit sehr hoch. Durch die Verwendung von Nanodrähten im Kontaktbereich wird die Kontaktfläche maßgeblich vergrößert (Abb. 7 rechts).
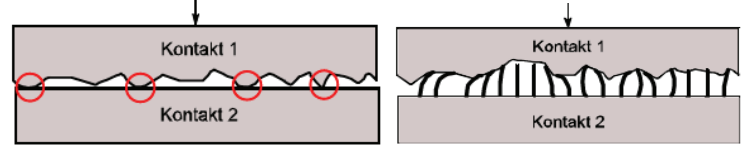

Abb. 7: Herkömmliche (links) und mit Nanodrähten versehene Kontaktflächen (rechts)

Die Nanodrähte schmiegen sich nahezu perfekt der Gegenfläche an. Durch diese Technologie kann bei gleicher Kontaktkraft der Kontaktwiderstand erheblich verringert werden [2].

Die Nanodrähte aus Gold werden durch optische Lithographie, galvanische Abscheidung und die Verwendung einer PolycarbonatTemplat hergestellt (Abb. 8). Der Ablauf der einzelnen Prozesse ist in [3] und [4] ausführlich beschrieben.

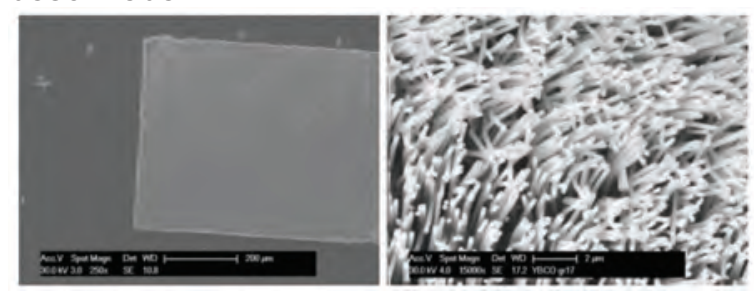

Abb. 8: REM-Aufnahmen (Übersicht links, im Detail rechts) von Gold-Nanodrähten[2]

Für die Untersuchungen der zu erwartenden Kontaktkräfte und der Montage des Gesamtsystems binärer Mikroschalter wurden verschiedene Kontaktflächendesign mit variierenden $\mathrm{Pa}$ rametern der Nanodrähte realisiert. Variiert

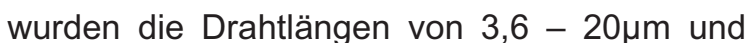
der Drahtdurchmesser von $100-200 \mathrm{~nm}$. Das Aspektverhältnis von 90:1 und die Dichte von 6 Nanodrähten auf $1 \mu \mathrm{m}^{2}$ blieben unverändert. Als optimal erwies sich eine Länge von $10 \mu \mathrm{m}$ und ein Drahtdurchmesser von $150 \mathrm{~nm}$, da die schlanken Drähte zu schnell knicken. Das Grundsubstrat ist Borosilikatglas. 


\section{Aufbau des Gesamtsystems}

In der Abb. 9 ist das Prinzip des Mikroschalters gezeigt. Im Zentrum befindet sich die Biegeplatte, welche mit dem Hydrogel das Unimorph bildet. Durch das Quellen unter Einfluss der Luftfeuchtigkeit bewegt sich das biegesteife Siliziumzentren (Centerboss) auf die mit Nanodraht bestückten Kontakte und schließt den elektrischen Kontakt bei erreichen des Schwelwertes.

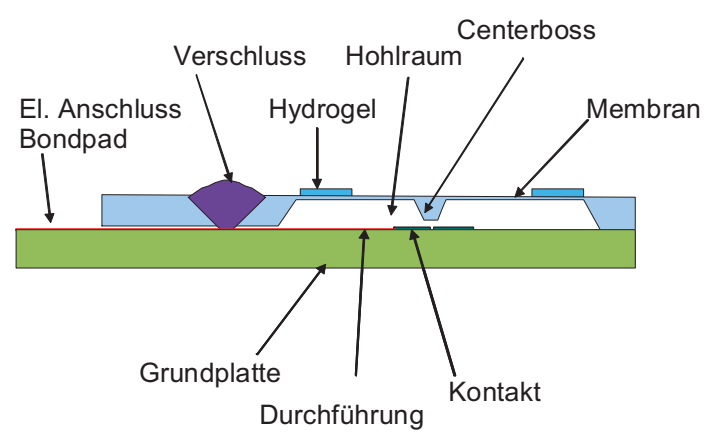

Abb. 9: Prinzip des aufgebauten Mikroschalters

Die elektrischen Anschlüsse werden mit Hilfe einer horizontalen Durchkontaktierung nach außen geführt. Gefügt werden die Grundplatte und Membranwafer durch anodisches Bonden. Die Abb. 10 zeigt den Waferverbund mit Sicht auf die Bondpads und die Rahmen für das Hydrogel.

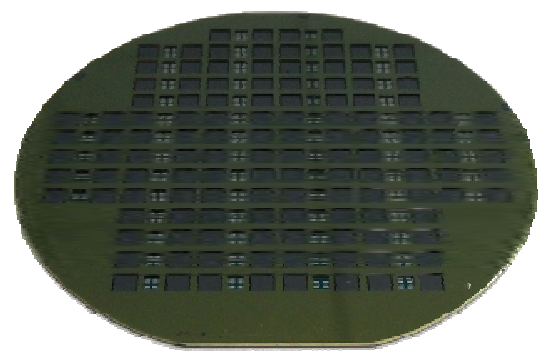

Abb. 10: Siliziumbiegeplatte mit Grundplatte im Waferverbund, anodisch gebondet

\section{Danksagung}

Die Arbeiten werden im Rahmen der finanziellen Unterstützung vom Bundesministerium für Bildung und Forschung (BMBF) (SEMIS, FKZ: 16SV5506K) durchgeführt.

\section{Referenzen}

[1] Th. Frank, G. Gerlach, A. Steinke, "Binary ZeroPower Sensors an alternative solution for powerfree energy-autonomous sensor systems", Microsyst Technol (2012) 18:1225-1231 DOI $10.1007 / \mathrm{s} 00542-012-1547-4$ 18.04.2012 20.04.2012.

[2] S. S Baek, R. S. Fearing, "Reducing contact resistance using compliant nickel nanowire arrays",

\section{Zusammenfassung und Anwendungsmög- lichkeiten}

Die Kombination der Sensorplattform mit den Spezialpolymerstrukturen und Nanotechnologien erlaubt ein neues Mikroschalterprinzip, dass die ständige Überwachung von Prozessen ermöglicht. Der herausragende Vorteil dieses variabel einsetzbaren Mikroschalters gegenüber anderen Lösungen ist, das eventabhängige und stromlose aktivieren von Sensorknoten aus dem Standby Zustand. Daher sind Batterielösungen in einer Vielzahl von Anwendungen ausreichend, da die Datenübertragung erst bei Bedarf, z.B. bei Erreichen eines Schwellwertes, eingeschaltet wird.

Eine Beispielanwendung ist die vielfach benötigte Messgröße „relative Luftfeuchte“. Sie hat große Bedeutung beim Erhalt der Qualität jeglicher Art von Gütern. Für alle Bereiche existieren Grenzwerte die zur Sicherung der Qualität, insbesondere über längere Zeiträume, nicht überschritten werden dürfen.

$\mathrm{Zu}$ den Anwendungen, sowohl Einzelanwendungen als auch Sensornetzwerke gehören:

- Die Überwachung der Genussreife von Lebensmitteln in der Lagerhaltung.

- Die Lager- und Transportüberwachung von feuchtigkeitsempfindlichen Gütern,

- Die Überwachung und Steuerung des Raumklimas, speziell in Lagerräumen, Museen, Archiven, Büchereien, Laboren, Rechenzentren und industriellen Produktionsanlagen.

- Der Einsatz als Feuchtewächter an unzugänglichen Stellen zur Verhinderung des Schimmelwachstums.

IEEE Transactions on Components and Packaging Technologies, Vol. 31, No. 4, 12.2008

[3] S. Quednau, H. F. Schlaak, "In-situ generation of arrays of metallic micro-and nanowires in silicon microsystems" - "In-Situ Erzeugung von Arrays aus metallischen Mikro- und Nanodrähten in Silizium-Mikrosystemenen", Mikro-Nano-Integration (GMM-FB 68) - 3. GMM-Workshop, Stuttgart, ISBN: 978-3-8007-3334-7, 03.2011.

[4] F. Greiner, S. Quednau, F. Dassinger, R. Sarwar, H. F. Schlaak, M. Guttmann, P. Meyer, Fabrication techniques for multiscale, 3D-MEMS with vertical metal micro- and nanowire integration, J. Micromech. Microeng. 23 (2013) 025018 (12pp) 